\title{
Subarachnoid Hemorrhage Caused by Rupture of a Distal Anterior Inferior Cerebellar Artery Aneurysm
}

\author{
-Three Case Reports-
}

\author{
Atsushi SAITO, Akiko NiSHINO, Ichiro SUZUKI, Hiroyasu SUZUKI*, \\ Akihiro UTSUNOMIYA, Shinsuke SUZUKI, \\ Hiroshi UENOHARA, and Yoshiharu SAKURAI \\ Department of Neurosurgery, Stroke Center, and \\ ${ }^{*}$ Department of Neuropathology, Sendai Medical Center, Sendai, Miyagi
}

\begin{abstract}
Three female patients, two under 35 years old, presented with ruptured aneurysms of the distal anterior inferior cerebellar artery (AICA) manifesting as subarachnoid hemorrhage. The first patient had a ruptured saccular aneurysm of the meatal loop of AICA, which was treated by direct neck clipping. The second patient had a ruptured aneurysmal lesion that arose from a microvascular anomaly of the dorsolateral portion of the AICA, which was successfully treated by trapping. The third patient was dead on arrival, and autopsy revealed a ruptured saccular aneurysm in the meatal loop of the AICA. The mechanism of development of distal AICA aneurysm remains unclear, and some cases indicate a complicated causal relationship between the aneurysms and vascular anomalies. Neurosurgeons need to carefully evaluate the vascular structure around the aneurysms by preoperative angiography in each case, and select the most appropriate strategy.
\end{abstract}

Key words: anterior inferior cerebellar artery, intracranial aneurysm, subarachnoid hemorrhage, vascular anomaly

\section{Introduction}

Aneurysms of the distal anterior inferior cerebellar artery (AICA) account for only $0.025 \%$ of all intracranial aneurysms. ${ }^{6,9,13,14,17)}$ The AICA is a branch of the basilar artery and courses inferolaterally toward the cerebellopontine angle, crosses the abducens nerve at the angle and the cerebellar flocculus between the facial and acoustic nerves. The meatal loop is located anterior to the flocculus and posterior to the auditory canal. Most AICA aneurysms (51\%) are located close to the branches of the internal acoustic artery in the meatal loop. ${ }^{6,9-11,15,17)}$ AICA aneurysms far distal to the meatal loop are exceedingly rare, and only $0.13 \%$ of all AICA aneurysms occur in the dorsolateral portion. Aneurysms in the AICA are 2.5 times more common in females than in males, and from 17 to 81 years old (mean 48.2 years old). ${ }^{8,11,13-15,17)}$ Only 84 cases of distal AICA aneurysm have been reported, and the distal AICA is the least likely intracranial main arterial trunk to harbor an aneurysm..$^{8,11,13-15,17)}$

Ruptured AICA aneurysms manifested as acute onset of subarachnoid hemorrhage (SAH) in $67 \%$ of cases, associated with sudden severe headache, meningismus, nausea, vomiting, photophobia, and sudden coma. ${ }^{8,11,13-15,17)}$ Another $20 \%$ of cases manifested as cerebellopontine angle syndrome, which consists of tinnitus, hearing loss, vertigo, facial paresis, gait ataxia, dysmetria, diplopia, and facial sensory disturbance, in patients with large aneurysms as a result of a mass effect rather than of hemorrhage. ${ }^{15,17)}$ These symptoms result from compression of cranial nerves or the brainstem, and may be prodromal signs of rupture. ${ }^{4,17)}$ The clinical and neuroimaging features of this unusual lesion have been discussed, but the pathological features and surgical issues have never been thoroughly exam-

Received October 13, 2007; Accepted July 9, 2008

Author's present address: Atsushi Saito, M.D., Department of Neurosurgery, Iwate Prefectural Central Hospital, Morioka, Iwate, Japan. 
ined.

We report three cases of distal AICA aneurysm manifesting as $\mathrm{SAH}$, and describe direct surgical observations of the vascular structures.

\section{Case Reports}

Case 1: A 20-year-old woman presented with sudden onset of severe headache. On admission, she had slight consciousness disturbance and lower cranial nerve paresis. No facial nerve or hearing impairment was detected. Computed tomography (CT) revealed SAH, predominantly in the left ambient cistern (Fig. 1). Cerebral angiography showed a 4-mm aneurysmal dilatation of the meatal loop of the left AICA (Fig. 2). Surgical exploration through the lateral suboccipital approach identified a saccular aneurysm arising from a branch of the meatal loop

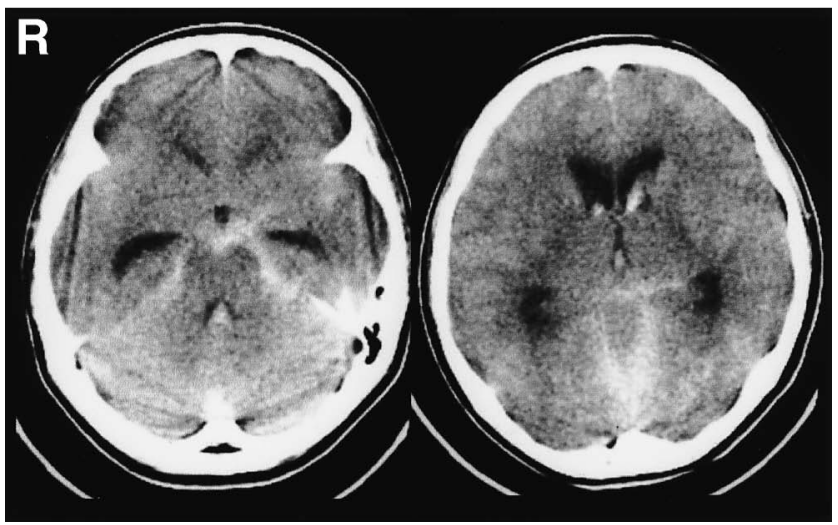

Fig. 1 Case 1. Computed tomography scans revealing subarachnoid hemorrhage predominantly located in the left ambient cistern.
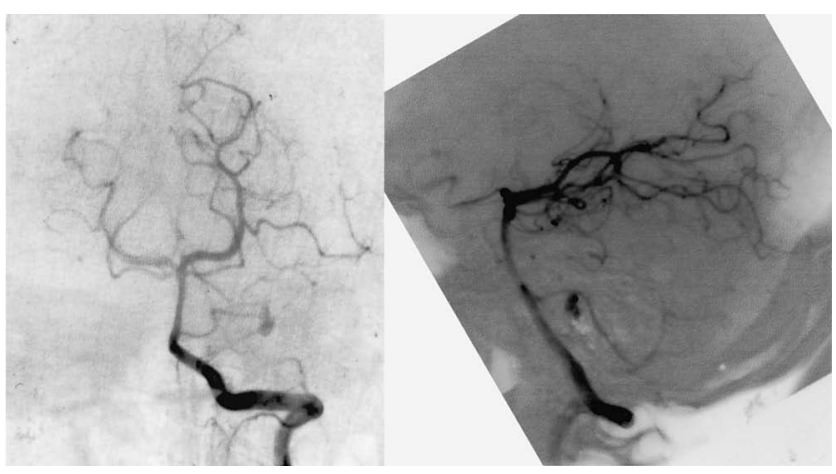

Fig. 2 Case 1. Left vertebral angiograms demonstrating a 4-mm aneurysmal dilatation in the meatal loop of the left anterior inferior cerebellar artery. of the AICA. The neck of the aneurysm was exposed and successfully clipped. After the surgery, her lower cranial nerve paresis gradually improved, and the patient recovered full neurological function by the time of discharge.

Case 2: A 31-year-old woman presented with sudden onset of severe headache without cranial nerve paresis or consciousness disturbance. CT on admission revealed thin $\mathrm{SAH}$ in the right quadrigeminal cistern and a thin subdural hematoma in the right posterior cranial fossa (Fig. 3). Cerebral angiography showed a $5-\mathrm{mm}$ aneurysmal dilatation in the dorsolateral portion of the right AICA (Fig. 4). Surgical exploration through a lateral suboccipital craniotomy showed the lesion arose from a vascular aggregation

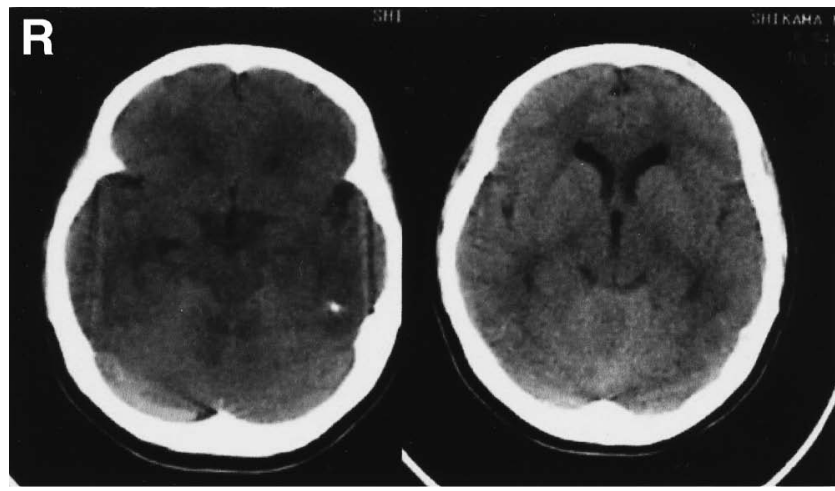

Fig. 3 Case 2. Computed tomography scans on admission demonstrating thin subarachnoid hemorrhage in the right quadrigeminal cistern and a thin subdural hematoma in the right posterior cranial fossa.

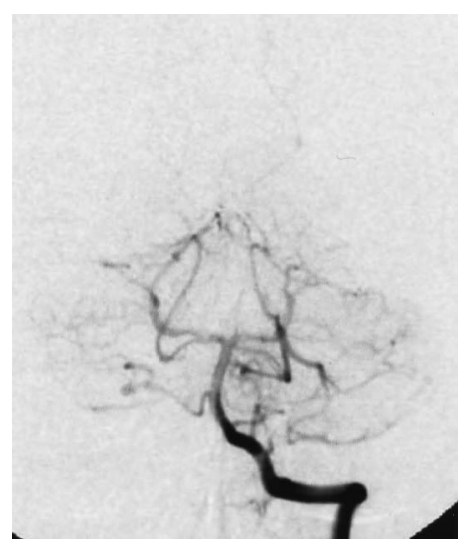

Fig. 4 Case 2. Left vertebral angiogram demonstrating a 5-mm aneurysmal dilatation in the dorsolateral portion of the right anterior inferior cerebellar artery. 
consisting of several small arteries in the lateral dorsal portion of the seventh and the eighth cranial nerves, without a simple neck (Fig. 5A). The lesion resembled the aneurysmal dilatation associated with a small arteriovenous malformation (AVM). We could not clearly identify the ruptured aneurysm. The vascular mass was trapped with two clips at the distal and proximal ends of the lesion and then was resected. Histological examination showed a vascular anomaly consisting of microvessels (Fig. 5B).

Postoperative angiography 2 weeks after the onset demonstrated enlargement of the remnant aneurysmal lesion compared with the preoperative aneurysmal lesion (Fig. 6). This was thought to be the untreated ruptured aneurysm. Therefore, the resected lesion was a dilated vascular anomaly located close to the untreated ruptured aneurysm, which was not observed at the first angiography. The ruptured aneurysm was approached by the same procedure as used during the first operation, and the clip placed at the first operation was used as a marker to locate the target lesion. The target ruptured lesion was located in the portion dorsal to the first clip. The ruptured aneurysm was located in the same vascular network of the first resected lesion but did not originate from the same branch. Exposure found a sphere-shaped dilatation of the vascular branch with some blebs. The lesion was trapped with a clip and resected (Fig. 7A). Histological examination revealed a defect in the medial layer of the artery (Fig. $7 \mathrm{~B})$. The patient was discharged without neurological deficit.

Case 3: A 64-year-old woman suffered onset of mild headache and nausea on awakening in the morning.

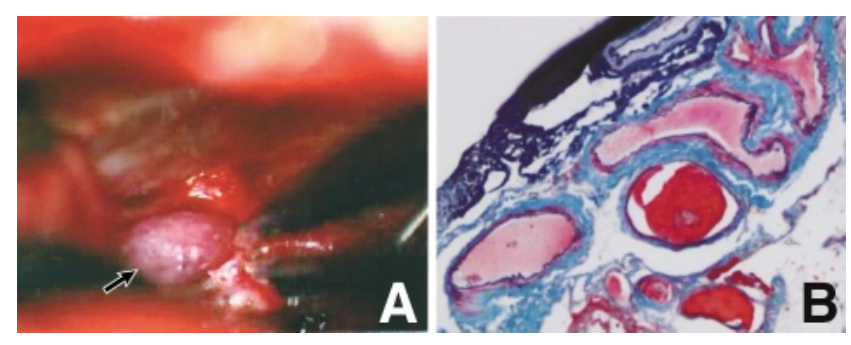

Fig. 5 Case 2. A: Intraoperative photograph during the first operation showing that the lesion arose from a vascular aggregation consisting of several small arteries without a simple neck (arrow). B: Photomicrograph of the resected aneurysmal dilatation of the distal anterior inferior cerebellar artery showing a microvascular anomaly consisting of microvessels. Elastica-Masson stain, $\times 40$.

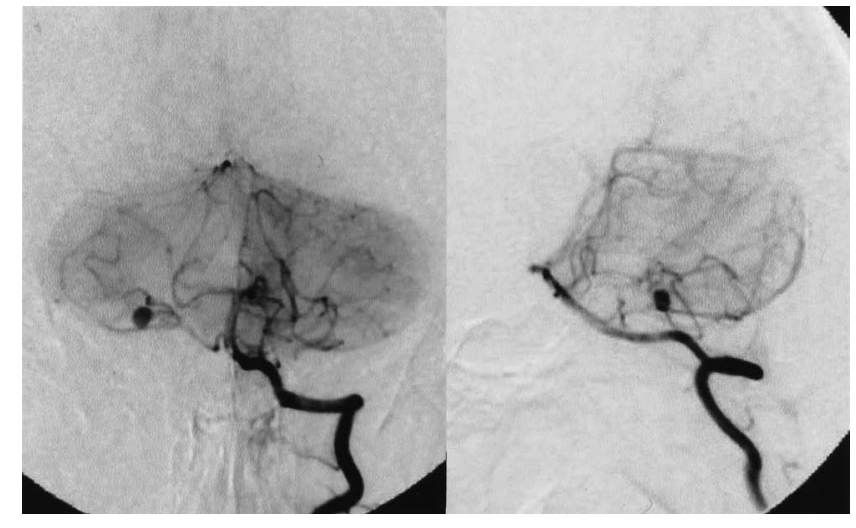

Fig. 6 Case 2. Postoperative left vertebral angiograms after the first operation showing enlargement of the remnant aneurysmal lesion at the distal anterior inferior cerebellar artery.
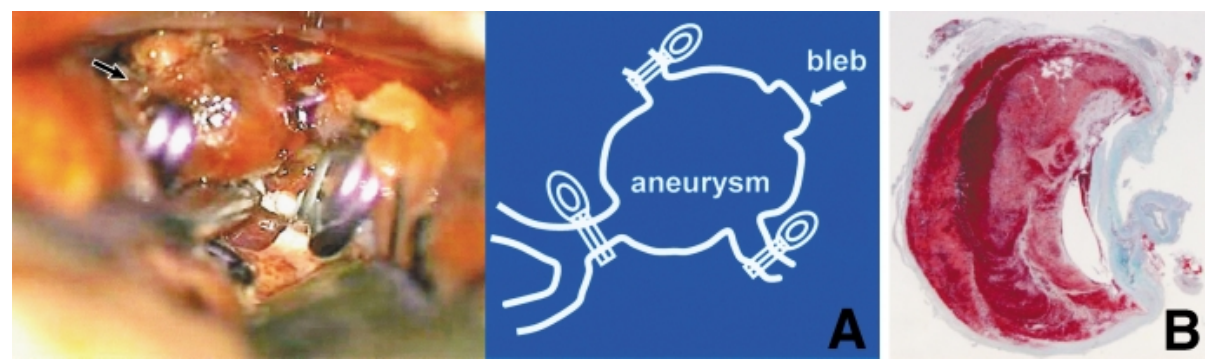

Fig. 7 Case 2. A: Intraoperative photograph during the second operation showing the ruptured aneurysm (arrow) adjacent to the site of resection of the aneurysmal lesion at the first operation. Illustrated scheme demonstrating the aneurysm trapped with a clip and resected. B: Photomicrograph of the resected specimen from the second operation revealing that the vascular lesion was a saccular aneurysm with a defect in the medial layer. Elastica-Masson stain, $\times \mathbf{4 0}$. 
She came to our department and soon lapsed into a comatose state. CT revealed a large SAH predominantly localized in the right side of the sylvian fissure and ambient cistern (Fig. 8). The patient died 5 hours after arrival. Autopsy demonstrated thick $\mathrm{SAH}$ in the basal cistern and a ruptured aneurysm arising from the meatal loop of the right AICA (Fig. 9A). Histological examination of the ruptured aneurysm revealed a saccular aneurysm, with a thin medial layer and a defect of the elastic layer in its wall (Fig. 9B).

\section{Discussion}

Our first patient had a ruptured saccular aneurysm

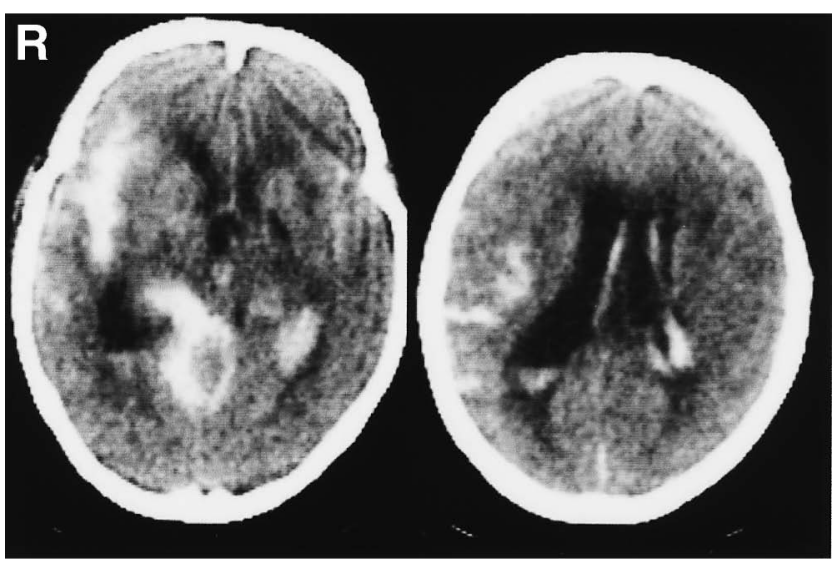

Fig. 8 Case 3. Computed tomography scans on arrival demonstrating thick subarachnoid hemorrhage, predominantly localized in the right side of the sylvian fissure and the ambient cistern. of the meatal loop of the AICA, which was treated by direct clipping. Our second patient had a ruptured aneurysmal lesion that arose from a microvascular anomaly in the dorsolateral portion of the AICA, which was successfully treated by trapping and resection. Our third patient was dead on arrival and autopsy revealed a ruptured saccular aneurysm of the meatal loop of the AICA. Our patients were all female and two were aged under 35 years. There were no warning signs of onset of SAH. Lateral suboccipital retrosigmoid craniotomy was performed in two cases and autopsy in one case to explore the lesions, and the vascular structure was observed directly.

The mechanism of development of distal AICA aneurysm is unknown, but aneurysms located on the distal side of the posterior cranial fossa, including aneurysms of the AICA and posterior inferior cerebellar artery, arise at both the branched and the curved portions of the arteries. The vertebrobasilar arteries consist of a reticular network of arteries in the fetal period, and the AICA gradually develops from this arterial network. ${ }^{1,4,5)}$ A gradual defect of a branch forms the curving portion of the AICA. This consecutive change of reticular network into simple curve of an artery may be related to the weakness of the arterial wall at the curve, which may be important in the development of aneurysms or aneurysmal vascular anomalies. The fetal development of the vertebrobasilar artery system may also affect the development of aneurysms associated with an anomalous vascular network or AVM in this region.

We could obtain histological findings of the aneurysms in our second and third cases. The ruptured vascular lesion was a saccular aneurysm associated with weakness of the arterial wall, occur-
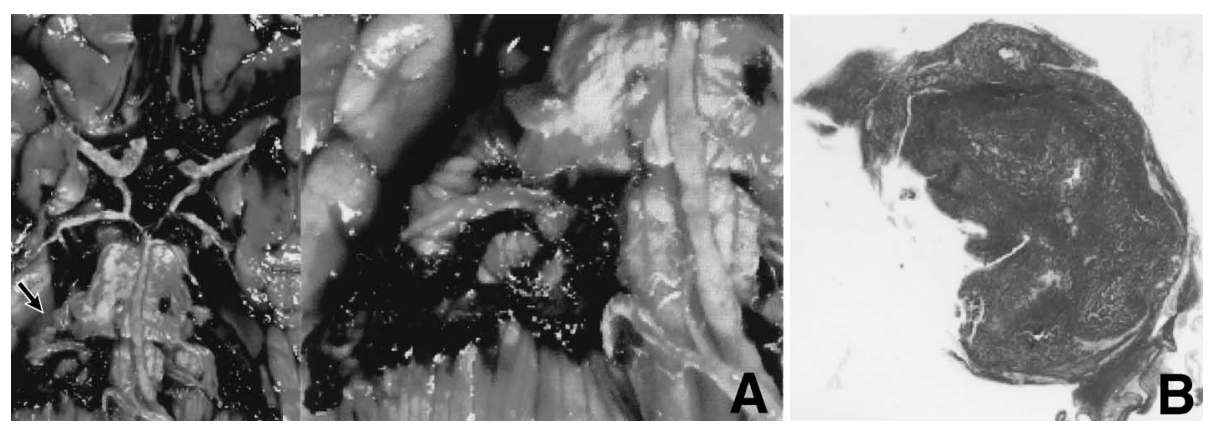

Fig. 9 Case 3. A: Photographs at autopsy showing thick subarachnoid hemorrhage localized in the basal cistern and a ruptured aneurysm at the meatal loop of the right anterior inferior cerebellar artery (arrow). B: Photomicrograph of the ruptured aneurysm revealing that the vascular wall of the saccular aneurysm had a thin medial layer and a defect in the elastic layers. Elastica-Masson stain, $\times \mathbf{4 0}$. 
ring as a defect of the medial layer or elastic layer, in both cases. Similar findings are obtained in the aneurysms of other intracranial arteries. However, the aneurysm was located in a very distal segment of the AICA in the second case, arose from an aggregation of small vessels associated with microvascular anomalies, and occurred as a sphere-shaped dilatation of the distal branch of the AICA. This finding may be related to the specific process of development of the vertebrobasilar artery system in the fetal period. ${ }^{8)}$ Moreover, the aneurysmal lesion did not have a single neck at the origin from the AICA. Such lesions may be not easy to completely treat by simple neck clipping, and the arterial circulation around the lesion may be difficult to preserve if resection is performed.

Among recent cases, postoperative facial nerve disturbance occurred in 1 of 13 cases and auditory disturbance in 4 of 8 cases of distal AICA aneurysm, despite the absence of such preoperative neural deficits. ${ }^{7)}$ The cause of the cranial nerve dysfunction was direct nerve injury or damage to the blood supply.7) Postoperative development of collateral flows around the internal acoustic artery prevented permanent nerve paralysis in some cases. However, if the neurosurgeon selects trapping to treat a distal AICA lesion, the collateral flow around the distal portion of the AICA may be difficult to evaluate preoperatively.

The mechanism of development of distal AICA aneurysms was discussed based on 6 cases associated with AVMs. ${ }^{10}$ Most AICA aneurysms were located proximal to the feeder of the AVM, and flowrelated hemodynamic stress caused by the AVM probably contributed to the formation of the distal AICA aneurysms. ${ }^{2,3,10,11)}$ Treatment of only the aneurysm or only the AVM in this region might lead to increased risk of bleeding from the remaining lesion because of the altered hemodynamics. ${ }^{10,12,13,16)}$ In our second case, the ruptured lesion was neither an AVM nor an aneurysm associated with AVM. The second angiography revealed enlargement of the aneurysm, possibly indicating that the ruptured lesion was located in the same vascular network as the resected vascular anomaly. Resection of the vascular anomaly close to the ruptured lesion might have caused hemodynamic change of the vascular network and increased hemodynamic stress on the ruptured aneurysm.

The mechanism of development of distal AICA aneurysms remains unclear, and a complicated causal relationship probably exists between the aneurysms and vascular anomalies, as in one of our present cases. The vascular structure around the aneurysms must be carefully evaluated by preopera- tive angiography in each case and the most appropriate strategy selected to prevent rebleeding.

\section{References}

1) Fujimura $N$, Abe T, Hirohata $M$, Morimitsu $H$, Tokutomi T, Shigemori M: Bilateral anomalous posterior inferior cerebellar artery-anterior inferior cerebellar artery anastomotic arteries associated with a ruptured cerebral aneurysm. Neurol Med Chir (Tokyo) 43: 396-398, 2003

2) Gacs G, Vinuela F, Fox AJ, Drake CG: Peripheral aneurysms of the cerebellar arteries. Review of 16 cases. J Neurosurg 58: 63-68, 1983

3) Guzman R, Grady MS: An intracranial aneurysm on the feeding artery of a cerebellar hemangioblastoma. J Neurosurg 91: 136-138, 1999

4) Ildan F, Göçer AI, Bağdatoğlu H, Uzuneyüpoğlu Z, Tuna M, Cetinalp E: Isolated trigeminal neuralgia secondary to distal anterior inferior cerebellar artery aneurysm. Neurosurg Rev 19: 43-46, 1996

5) Kaech D, de Tribolet N, Lasjaunias P: Anterior inferior cerebellar artery aneurysm, carotid bifurcation aneurysm, and dural arteriovenous malformation of the tentorium in the same patient. Neurosurgery 21: 575-582, 1987

6) Kamano S, Kirino $\mathrm{T}$, Mizuno S: Intrameatal aneurysm. Neurochirurgia (Stuttg) 29: 28-30, 1986

7) Kamiya K, Nagai H, Koide K, Yamashita N, Shimazu N: Peripheral anterior inferior cerebellar artery aneurysms. Surg Neurol 42: 46-51, 1994

8) Kondoh T, Kurihara E, Kohmura E: Distal anterior inferior cerebellar artery aneurysm occult on magnetic resonance angiography one month prior to rupture. Neurol Med Chir (Tokyo) 43: 345-348, 2003

9) Locksley HB: Natural history of subarachnoid hemorrhage, intracranial aneurysms and arteriovenous malformations. Based on 6368 cases in the cooperative study. J Neurosurg 25: 219-239, 1966

10) Menovsky T, Andre Grotenhuis J, Bartels RH: Aneurysm of the anterior inferior cerebellar artery (AICA) associated with high-flow lesion: report of the two cases and review of literature. J Clin Neurosci 9: 207-211, 2002

11) Mizushima H, Kobayashi N, Yoshiharu S, Kazuo H, Dohi K, Sasaki K, Matsumoto K: Aneurysm of the distal anterior inferior cerebellar artery at the medial branch: a case report and review of the literature. Surg Neurol 52: 137-142, 1999

12) Nishi S, Taki W, Nakahara I, Yamashita K, Sadatoh A, Kikuchi $\mathrm{H}$, Hondo $\mathrm{H}$, Matsumoto $\mathrm{K}$, Iwata $\mathrm{H}$, Shimada Y: Embolization of cerebral aneurysms with a liquid embolus. EVAL mixture: report of three cases. Acta Neurochir (Wien) 138: 294-300, 1996

13) Oana K, Murakami T, Beppu T, Yamaura A, Kanaya $\mathrm{H}$ : Aneurysm of the distal anterior inferior cerebellar artery unrelated to the cerebellopontine angle: case report. Neurosurgery 28: 899-903, 1991

14) Saito A, Ezura M, Takahashi A, Yoshimoto T: [An 
arterial dissection of the distal anterior inferior cerebellar artery treated by endovascular therapy]. No Shinkei Geka 28: 269-274, 2000 (Jpn, with Eng abstract)

15) Saito R, Tominaga T, Ezura M, Shimizu H, Yoshimoto $\mathrm{T}$ : [Distal anterior inferior cerebellar artery aneurysms: report of three cases and literature review]. No Shinkei Geka 29: 709-714, 2001 (Jpn, with Eng abstract)

16) Vincentelli F, Caruso G, Licastro G, Rabehanta P: Association between an aneurysm of the anterior inferior cerebral artery and an arteriovenous malformation fed by the same artery. J Neurosurg Sci 42:
41-45, 1998

17) Zager EL, Shaver EG, Hurst RW, Flamm ES: Distal anterior inferior cerebellar artery aneurysms. J Neurosurg 97: 692-696, 2002

Address reprint requests to: Atsushi Saito, M.D., Department of Neurosurgery, Iwate Prefectural Central Hospital, 1-4-1 Ueda, Morioka, Iwate 020-0066, Japan.

e-mail: satsushi2002@yahoo.co.jp 